\title{
Thermal Conductivity Reduction in Analogous 2D Nanomaterials with Isotope Substitution: Graphene and Silicene
}

\author{
Srilok Srinivasan ${ }^{1}$, Upamanyu Ray ${ }^{1}$ and Ganesh Balasubramanian ${ }^{1,2^{*}}$ \\ ${ }^{1}$ Department of Mechanical Engineering and ${ }^{2}$ Microelectronics Research Center, Iowa \\ State University, Ames, IA 50011, USA
}

\begin{abstract}
We employ molecular dynamics simulations to understand how presence of isotopes influences thermal transport across silicene, and compare the findings with that in structurally analogous graphene. The simulated structures are about $140 \mathrm{~nm}$ long and around $4 \mathrm{~nm}$ wide. The phonon spectra along with the variation of thermal conductivity reveal that out-of-plane modes are delocalized relative to the in-plane counterparts. The absolute thermal conductivity reductions are more pronounced in graphene than in silicene. Our computational findings agree with results of an analytical model based on mean-field approximation with appropriate corrections for the lattice anharmonicity.
\end{abstract}

\section{Keywords}

Isotope substitution $\bullet$ thermal conductivity $\bullet$ phonon density of states $\bullet$ silicene $\bullet$ graphene $\bullet$ molecular dynamics $\bullet$ mean-field approximation

${ }^{*}$ Corresponding author

Department of Mechanical Engineering, 2092 Black Engineering, Iowa State University, Ames, IA 50011, USA. Phone: +1 -515-294-9226, Email: bganesh@iastate.edu 


\section{Introduction}

Thermal transport in graphene, a planar sheet of $\mathrm{sp}^{2}$-bonded carbon atoms in a honeycomb lattice, has been extensively investigated over the last decade using both experimental ${ }^{1-6}$ and theoretical ${ }^{7-10}$ approaches. In addition to the high thermal conductivities that are attractive for thermal management applications ${ }^{11,12}$, graphene also has novel electronic and optical properties due to the presence of Dirac cones ${ }^{13,14}$. On the other hand, defect engineered graphene nanostructures are potential candidates for fabricating thermoelectric devices with reduced thermal conductivity ${ }^{15}$. Analogous to the planar graphene, a stable hexagonal arrangement of silicon atoms in a two-dimensional (2D) structure, with local buckling ${ }^{16-18}$, termed as silicene, has been increasingly gaining attention ${ }^{19-21}$ due to its high crystallinity ${ }^{19,20,22,23}$, tunable band gaps ${ }^{24-26}$ as well as low thermal conductivity ${ }^{27,28}$ that make it an attractive nanomaterial for optoelectronics, transistor applications and efficient thermoelectric devices. Freestanding silicene has been shown to possess electronic and optical properties analogous to the massless Dirac fermions in graphene ${ }^{18}$. Moreover, unlike graphene it is easier to tune the band gap in silicene, for example, with the help of an external electric field ${ }^{25,26}$. Given the billions of dollars invested in establishing the silicon industry, if silicene can be enabled to produce remarkable transport properties for electronic and thermoelectric applications, one can envision a transformative progress in the area of semiconductors. However, before employing these materials in devices, a thorough understanding of heat conduction and transport properties is essential for constructing robust solutions for thermal management.

Thermal transport in both these 2D nanomaterials is known to occur predominantly by lattice vibrations (or phonons). While graphene is perfectly planar and has a hexagonal honeycomb lattice, both theoretical ${ }^{16-18,29,30}$ and experimental investigations ${ }^{19,22}$ show that silicene structure is buckled in the direction normal to the 2D plane. This structural difference between graphene and silicene leads to different phonon transport mechanisms resulting in different heat transfer characteristics ${ }^{31}$. The few fundamental investigations in the literature ${ }^{27,28,32}$ offer inconsistent predictions about the phonon modes exerting dominant contribution to heat transfer through silicene, emphasizing the need for further analyses of the underlying mechanisms driving the energy transport in silicene as 
compared to graphene. Two major schools of thought include those who suggest that, similar to graphene, heat transfer through silicene is dominated by out-of-plane flexural modes ${ }^{27}$, while an alternate theory ${ }^{31,32}$ proposes that the out-of-plane lattice vibrations contribute negligibly to the thermal transport in silicene and heat conduction occurs predominantly due to the in-plane modes.

The complexity is increased if there is mass disorder in these materials due to presence of defects of different forms, such as isotopes, vacancies, dopants etc. Correlating the thermal conductivity variation with the changes in the phonon spectra due to these defects is nontrivial and necessary to thoroughly characterize the thermal transport in silicene. Presence of isotopes in a material structure induces mass disorder, reducing phonon thermal conductivity $(k)$ due to enhanced energy scattering for even the smallest defect concentration. The reductions in thermal conductivity for isotope substituted carbon and silicon nanomaterials have been discussed in the literature ${ }^{33-37}$. Here, we employ molecular dynamics (MD) simulations to understand the physics of heat transfer in silicene, relative to that in graphene, by examining the role of different vibrational modes in both the pure and isotope substituted nanomaterials. Of the various isotopes of carbon and silicon, we substitute the ${ }^{12} \mathrm{C}$ and ${ }^{28} \mathrm{Si}$ atoms in the corresponding $2 \mathrm{D}$ structures with ${ }^{14} \mathrm{C}$ and ${ }^{30} \mathrm{Si}$ respectively. While ${ }^{14} \mathrm{C}$ is the longest living and heaviest of all the naturally occurring carbon isotopes, ${ }^{30} \mathrm{Si}$ is a very stable atom of silicon ${ }^{38}$. Our results, as elaborated below, show that the high frequency in-plane vibrational modes contribute in reducing heat transport through isotope substituted graphene, while the relatively less significant changes in phonon characteristics of silicene results in only small thermal conductivity variations. The predicted thermal conductivity reductions exhibit a similar trend, which is suitably represented by a mean-field approximation model corrected for the lattice anharmonicity.

\section{Computational methods}

MD simulations are employed to investigate thermal transport in silicene and graphene. The dimensions of graphene and silicene sheets are $140.24 \mathrm{~nm} \times 4.68 \mathrm{~nm}$ and $134.70 \mathrm{~nm}$ $\times 4.19 \mathrm{~nm}$ respectively, with the longer dimension being the armchair direction. The $z-$ 
axis represents the out-of-plane direction and the in-plane axes are along the $x$ - and $y$ directions. The $\mathrm{Si}-\mathrm{Si}$ bond length in silicene, as derived from earlier first principle calculations, is $0.2242 \mathrm{~nm}^{32}$ while the C-C bond in graphene is $0.1420 \mathrm{~nm}$ long. Due to the difference in bond lengths and the atomic diameters of $\mathrm{Si}$ and $\mathrm{C}$, the number of atoms in silicene and graphene are notably different even though their dimensions are similar. Graphene is one atom thick with the van der Waals diameter of $0.34 \mathrm{~nm}$ for $\mathrm{C}$, while the thickness of silicene is the sum of the height of buckling and van der Waals diameter of $\mathrm{Si}(0.42 \mathrm{~nm})$, as shown in Fig. 1. We randomly substitute ${ }^{12} \mathrm{C}$ and ${ }^{28} \mathrm{Si}$ atoms in the respective nanomaterials with their heavier counterparts, ${ }^{14} \mathrm{C}$ isotope in case of graphene and ${ }^{30} \mathrm{Si}$ for silicene.

The dimension of the simulation box in the $x$ - and $y$-directions corresponds to the length and width of the sheets, respectively. Periodic boundary conditions (PBCs) are imposed in all directions. Since the system is surrounded by the periodic images along the plane of the material, there are no unsaturated bonds on the edges. The $z$-wise dimensions of the simulation box are kept substantially large $(\sim 20 \mathrm{~nm})$ to prevent the nanomaterials from interacting with their own periodic images along this direction. The interactions between $\mathrm{C}-\mathrm{C}$ atoms in graphene are modeled using optimized Tersoff potential for thermal transport in graphene ${ }^{39,40}$. However, when the Tersoff model was used for $\mathrm{Si}-\mathrm{Si}$ interactions in silicene, the structure did not show any buckling. In order to account for the buckling of the silicene structure we employ the recently optimized Stillinger Weber (SW) potential parameters ${ }^{32}$ to model the interaction between Si atoms. To the best of our knowledge, this is the only available set of SW potential parameters that reproduce the low buckling structure of silicene as well as generate accurate phonon dispersion curves. All the simulations are performed with the highly parallelized LAMMPS package ${ }^{41}$ and VMD tools are used for the visual analyses ${ }^{42}$.

Though silicene has a low buckling, we initialize our simulations with a perfectly planar honeycomb lattice for both graphene and silicene. The geometry of each structure is optimized by energy minimization. The resulting energy-minimized structures are shown in Fig. 1. While graphene retains the planar structure, silicene demonstrates buckling with 
$h=0.04272 \mathrm{~nm}$, in agreement with earlier reports ${ }^{18,26,32}$ validating our choice of the potential model. The optimized structures are then equilibrated at a temperature of $300 \mathrm{~K}$ and at a pressure of 0 bar under NPT ensemble for 4 ns using the Nosé-Hoover thermostat and barostat, each with a coupling constant of $0.1 \mathrm{ps}$. This is followed by $4 \mathrm{~ns}$ of equilibration in canonical (NVT) ensemble, using the Nosé-Hoover thermostat with coupling constant of $0.1 \mathrm{ps}$. The system is subsequently simulated without any constraints under the NVE ensemble for 2 ns to ensure energy conservation. The time step used in all our simulations is $1 \mathrm{fs}$.

Upon equilibration, we compute the mass-weighted velocity autocorrelation function (VACF) from the trajectories over the next $1 \mathrm{~ns}$. The vibrational density of states (DOS) is obtained by considering the Fourier transform of VACF, as represented by

$$
P(\omega)=\frac{1}{\sqrt{2 \pi}} \int_{0}^{\infty} e^{i \omega t}\left|\sum_{j=1}^{N} m_{j} v_{j}(t) \cdot m_{j} v_{j}(0)\right| d \omega
$$

where $\omega$ is the frequency of vibration, $m_{j}$ and $v_{j}$ are the mass and velocity of atom $j$. Thermal conductivity, $k$, is computed using the reverse non-equilibrium MD (RNEMD) technique by employing the Müller-Plathe method ${ }^{43}$ with single kinetic energy swaps every $300 \mathrm{fs}$ and the system divided into 100 bins along the direction of heat flow. The kinetic energy swaps are done for $7 \mathrm{~ns}$ before sampling the data for determining the temperature gradient in order to ensure that the system has reached steady state and a linear temperature profile is obtained. It is important to note that for the cross-section area used in the $k$ calculation, we simply consider a rectangular cross section of width $(y$ axis) equal to that of graphene or silicene, and thickness equal to the van der Waals diameter of the $\mathrm{C}$ in the case of graphene, and the sum of the van der Waals diameter of $\mathrm{Si}$ and height of undulations in the case of silicene. Although all our MD calculations are at $300 \mathrm{~K}$, which is much below the Debye temperature of graphene $\left(\sim 2100 \mathrm{~K}^{11}\right)$, the use of classical thermostats in equilibration process is justified since the relative change in thermal conductivity with isotope concentration at a fixed temperature remains unaffected even after quantum corrections. In addition, it has been shown that the difference between the thermal conductivity calculated using quantum corrections and the classical thermal conductivity at $300 \mathrm{~K}$ and above are within an acceptable range ${ }^{44}$. The 
errors in the predictions are obtained by applying ergodic hypothesis and computing $k$ at different time intervals once steady state is attained.

\section{Results and Discussion}

Phonon density of states in isotope-substituted graphene: Thermal transport in 2D nanomaterials is due to the three acoustic phonon modes: in-plane longitudinal mode along $x$ - direction, in-plane transverse mode along $y$ - direction and out-of-plane flexural mode in the $z$-direction. We examine the phonon spectra of the in-plane modes and the out-of-plane flexural modes for $0 \%, 10 \%, 25 \%, 30 \%, 35 \%, 50 \%, 60 \%, 80 \%, 90 \%$, and $100 \%$ of isotope concentration. For the sake of clarity, we show only the DOS curves for 0\%, 25\%, 50\%, 80\%, and 100\% isotope substitution in Fig. 2. The DOS curves for graphene sheets along the $x$-, $y$-, and $z$ - directions are presented Figs. $2 \mathrm{a}, 2 \mathrm{~b}$, and $2 \mathrm{c}$ respectively. Along both the longitudinal $(x)$ and transverse $(y)$ in-plane directions, the phonon spectra presented in Figs. $2 \mathrm{a}$ and $2 \mathrm{~b}$ shows that the prominent vibrational modes along the in-plane directions are high frequency localized modes, with peaks at around 50 Terahertz (THz) for pure ${ }^{12} \mathrm{C}$ graphene sheets. On the other hand, the DOS spectra perpendicular to the direction of heat transfer in the out-of-plane direction peaks at much lower phonon frequencies of $11 \mathrm{THz}$ and $25 \mathrm{THz}$ for pure ${ }^{12} \mathrm{C}$ graphene sheet as shown in Fig. 2c.

Isotope substitution induces mass disorder in the lattice that results in increased phonon scattering at the defective sites due to the difference in the characteristic frequencies. The effect of mass disorder is very conspicuous for the vibrational modes in all three directions. With increasing isotope fraction from $0 \%$ to $100 \%$, there are two effects that can be observed from Figs. 2a, 2b, and 2c: (i) The frequencies of the vibrational modes are shifted to lower values (redshift) ${ }^{27}$ with increasing isotope fraction and (ii) the intensity of peaks are reduced as the extent of mass disorder is increased in the lattice. The redshift of DOS can be explained by the increase in the effective mass with the inclusion of heavier isotopes. Under harmonic approximation, the frequency of vibration

is proportional to $m_{\text {effective }}^{-0.5}$. The reduction in the peak intensities can be attributed to the mass disorder in the system. The mass disorder increases for isotope substitution from 
$0 \%$ to $50 \%$, lowering the intensity of the DOS peaks. The thermal conductivity reduces with increasing mass disorder as shown later in Fig. 4. When the ${ }^{14} \mathrm{C}$ concentration is more than $50 \%$, the isotope becomes the major component in the lattice and the mass disorder decreases with further substitution. Beyond 50\% isotope substitution, the DOS continues to shift to lower frequencies but the intensity of the peaks increases, simultaneously improving thermal conductivity (Fig. 4). Though the flexural modes have lower energies relative to the in-plane counterparts, we observe that the peak intensities are less affected by presence of isotopes in the lattice. Hence, the rather large reduction (around 70\%) in thermal conductivity of graphene (Fig. 4) can be attributed to the changes in the phonon characteristics of the short ranged in-plane modes. This also suggests that, though out-of-plane modes are relatively stiff ${ }^{46-48}$ and delocalized in nature, the role of in-plane modes in heat conduction in graphene cannot be neglected.

Phonon density of states in isotope substituted silicene: A similar analysis is performed for silicene to understand if any differences exist in its heat conduction mechanisms relative to that of graphene. Although graphene and silicene are analogous 2D materials, their structural configurations are not exactly similar with silicene deviating from the perfect planar lattice. The phonon DOS for silicene along the $x$-, $y$-, and $z$-directions are shown in Figs. 3a, 3b, and 3c. The vibrational spectra along the in-plane directions for pure silicene shows peak intensities around frequencies of $15.6 \mathrm{THz}$ and $5 \mathrm{THz}$ for both the longitudinal i.e. $x$-wise modes (Fig. 3a) as well as transverse modes i.e. $y$-wise (Fig. $3 b)$. However, in the out-of-plane direction, we observe only a single peak around a very low frequency of $\sim 1.2 \mathrm{THz}$.

The influence of mass disorder in shifting the vibrational spectra in silicene follows similar trend as in graphene. Figs. $3 \mathrm{a}, 3 \mathrm{~b}$ and $3 \mathrm{c}$ explicitly reveal that $25 \%, 50 \%, 80 \%$, and $100 \%$ isotope substitution in silicene lower the characteristic frequencies of the phonon modes along all the three directions due to increase in effective mass. However, the effect of mass disorder on the peak intensities is almost non-existent in silicene and the corresponding reduction in the thermal conductivity is very small (Fig 4). The minor reductions in the peak intensities with inclusion of mass disorder is recovered for isotope 
concentration of more than $50 \%$ wherein the extent of mass disorder decreases with further increase in isotope substitution. This, as shown in Fig. 4, reflects in the increasing thermal conductivity of the extensively $(>50 \%)$ isotope substituted nanomaterials, exhibiting a trend similar to graphene. In addition, like graphene, majority of the delocalized low frequency phonons in silicene are out-of-plane modes. However, the buckled structure of silicene significantly changes phonon lifetime as well as the harmonic and anharmonic force constants compared to a perfectly planar silicon honeycomb lattice ${ }^{49}$. The very low $k$ of silicene is due to its buckled nature ${ }^{31}$. Earlier reports show that silicene approaches a planar structure with application of tensile strain, and consequently thermal conductivity increases ${ }^{27,28,49}$. In this regard, the phonon scattering mechanisms in silicene are different from the perfectly planar graphene. We note the non-zero intensity at zero frequency in the DOS curves of silicene and the outof-plane DOS of graphene. This occurs because of a numerical approximation as our computations are based on discrete values obtained from MD simulations, and the very low frequency modes (between $0 \mathrm{THz}$ and $0.06 \mathrm{THz}$ ) are binned to the intensity at near zero frequency.

Thermal conductivity variation with isotope substitution: We show in Fig. 4 that with increase in isotope fraction $(\phi), k$ initially decreases until the isotope concentration is $50 \%$ where mass disorder is maximum. Beyond $\phi=0.5, k$ again increases representing the characteristic U-shaped curve ${ }^{28,33,45}$. The value of $k$ for graphene and silicene of the simulated dimensions composed of pure ${ }^{12} \mathrm{C}$ and pure ${ }^{28} \mathrm{Si}$ atoms are $577.55 \pm 41.70$ $\mathrm{W} / \mathrm{m} . \mathrm{K}$ and $11.14 \pm 0.05 \mathrm{~W} / \mathrm{m} . \mathrm{K}$ respectively, which are in agreement to the values reported for graphene and silicene of similar dimensions in earlier MD based investigations ${ }^{32,50}$. Increasing the fraction of isotopes from zero to 0.5 induces a gradual increase in mass disorder. Atoms in the lattice are either the lighter ${ }^{12} \mathrm{C}$ (for graphene) and ${ }^{28} \mathrm{Si}$ (for silicene) or correspondingly the heavier ${ }^{14} \mathrm{C}$ and ${ }^{30} \mathrm{Si}$. The vibrational frequencies of the 2D nanomaterial shift to lower values with increasing isotope fraction as discussed in Figs. 2 and 3. Additionally, the characteristic vibrational frequencies of the heavier and lighter masses are different. When both of the isotopes are present in a lattice, phonons of different frequencies interact. $k$ decreases till it reaches a minimum at 
$50 \%$ isotope substitution. While the frequencies continue to shift to lower values with further increase of isotope concentration, in contrast $k$ increases for $0.5<\phi<1.0$. For isotope concentration between $50 \%$ and $100 \%$, the heavier atoms comprise the majority of the 2D lattice structure. As mass disorder decreases, heat conduction is improved, as reflected in the increasing values of $k$. We observe that inclusion of a low fraction of mass disorder induces a relatively significant reduction in $k$, whereas the variations in thermal conductivity are relatively smaller for further increases in isotope concentration. This drastic decrease in $k$ for low isotope percentage is more prominent in graphene than in silicene. Likewise, we observe a stronger absolute reduction in $k$ for graphene though the trend of relative thermal conductivity decrease is similar for both nanomaterials.

Thermal conductivity predictions from mean-field approximation: We compare our $k$ reduction predictions with a classical model based on mean-field approximation ${ }^{45}$ which assumes that the heat transfer rate is proportional to the frequency, $\omega_{x}$ of a oscillator formed by connecting two different point masses, $M_{\alpha}$ and $M_{\beta}$. It follows from the meanfield approximation that the heat transfer reduction $k(\phi)$ can be given as ${ }^{45}$ :

$$
k(\phi)=k_{\phi} / k_{0} \sqrt{\frac{\varepsilon(1+\varepsilon)\left[(1-\phi+\varepsilon) M_{\alpha}+(\phi+\varepsilon) M_{\beta}\right]}{(\phi+\varepsilon)(1-\phi+\varepsilon)\left[(1+\varepsilon) M_{\alpha}+\varepsilon M_{\beta}\right]}},
$$

where $k_{\phi}$ is the thermal conductivity of isotope substituted nanomaterial, $k_{0}$ is the thermal conductivity of the pure nanostructure, $\phi$ is the molar fraction of the isotope $\beta$ that replaces atom $\alpha$ in the nanomaterial and $\varepsilon$ is a dimensionless numerical parameter to prevent divergence for the limiting cases. In a completely harmonic mean-field approximation the parameters $\varepsilon$ and $M_{\alpha} / M_{\beta}$ are determined using the following two boundary conditions: (1) $k(1)=\left(m_{\beta} / m_{\alpha}\right)^{-1 / 2}$ where $m_{\beta}$ and $m_{\alpha}$ are the atomic mass of isotope $\beta$ and $\alpha$ respectively, (2) $k(x)$ obtained from ${ }^{45}$

$k(x)=\frac{1}{\sqrt{x\left(m_{\beta} / m_{\alpha}\right)+(1-x)}}\left[x^{2}+(1-x)^{2}-x(1-x)\right]$

However, the predictions of this model compare poorly with simulation results of silicene when the mean field parameters are determined using the above-mentioned boundary conditions due to the anharmonicity in the system. It should be noted that the mean-fieldapproximation is based on a simple harmonic model and there will be deviations from it 
as the anharmonicity in the system increases. The buckled form of silicene induces considerable anharmonicity compared to the graphene structure. To predict the $k$ values accurately we need to apply necessary corrections to the mean field parameters; we use the following boundary conditions: $k(1)=k_{100} / k_{0}$ and $k(0.5)=k_{50} / k_{0}$ where $k_{100}, k_{50}$ and $k_{0}$ are the thermal conductivity values computed from MD simulations. The $k$ reduction with $\phi$ in equation (1) is presented in Fig. 4 for both graphene and silicene. The values of the parameters, $\varepsilon$ and $M_{\alpha} / M_{\beta}$, for both graphene and silicene are provided in Fig. 4. The model predictions compare very well with the results calculated from the atomistic simulations. While remarkable qualitative agreement is observed, the minor quantitative differences exist due to the limited sampling data used for computing $k$ values, instead of averaging over a series of calculations with different spatial isotope arrangements. The mean-field approximation model reproduces the significant decrease in $k$ for low isotope fraction and the relatively lower reduction when the isotope substitution is between $30 \%$ and $70 \%$ for both the materials. Hence, if the thermal conductivity values for $0 \%, 50 \%$, and $100 \%$ isotope substitution are only known, we can predict the approximate thermal conductivity for any isotope fraction using the mean field approximation. The predictions of the model, which requires only the masses of the atoms and fraction of isotopes for the isotope substituted graphene and silicene, enable us to corroborate that it is the mass disorder and different effective masses of $\mathrm{C}$ and $\mathrm{Si}$ atoms in respective nanomaterials that is responsible for the reduction in thermal conductivity.

\section{Conclusions}

In summary, our computational investigation employing molecular dynamics shows that, in presence of isotopes that induces a mass disorder in the structure, thermal conductivity reductions in these nanomaterials is ascribed essentially to the perturbation of the phonon modes. The phonon DOS in all the three directions suffers a redshift with increase in the effective mass due to the inclusion of isotopes. The reduction in thermal conductivity in silicene due to the isotope substitution is small compared to the reduction observed in graphene. While out-of-plane flexural modes are significantly stiffer and delocalized, the contribution of in-plane phonon modes to the overall thermal conductivity reduction in graphene cannot be neglected. Qualitatively, the variations of $k$ with increasing isotope fractions are similar in both graphene $(140.24 \mathrm{~nm} \times 4.68 \mathrm{~nm})$ and silicene $(134.70 \mathrm{~nm} \times$ 
$4.19 \mathrm{~nm}$ ). A mean field approximation based on harmonic interactions, appropriately corrected for the lattice anharmonicity, simply employs the masses and fractions of the two isotopes to predict the $k$ variations. The model results are in strong agreement with our atomistic simulations. Further efforts based on extensive theoretical investigations as well as experiments are required to conclusively determine the dominant phonon modes for heat conduction in silicene and how that varies with isotope substitution.

\section{Acknowledgement}

The work was supported by the National Science Foundation (NSF) grant no. CMMI1404938. We thank Dr. James Coyle, Director of High Performance Computing Operations, for help with the HPC cluster CyEnce, purchased through NSF MRI grant number CNS 1229081 and NSF CRI grant number 1205413, that was used for all the simulations. We thank Dr. Frédéric Leroy of Technische Universität Darmstadt for his valuable input in interpreting the DOS spectra. We also thank Dr. Alexander Balandin, University of California at Riverside, for constructive suggestions on improving the manuscript. 


\section{References}

1 S. Ghosh, I. Calizo, D. Teweldebrhan, E. P. Pokatilov, D. L. Nika, A. A. Balandin, W. Bao, F. Miao, and C. N. Lau, Applied Physics Letters 92, 151911 (2008).

2 J. H. Seol, I. Jo, A. L. Moore, L. Lindsay, Z. H. Aitken, M. T. Pettes, X. Li, Z. Yao, R. Huang, D. Broido, N. Mingo, R. S. Ruoff, and L. Shi, Science 328, 213 (2010).

A. A. Balandin, S. Ghosh, W. Bao, I. Calizo, D. Teweldebrhan, F. Miao, and C.

N. Lau, Nano Letters 8, 902 (2008).

K. M. F. Shahil and A. A. Balandin, Nano Letters 12, 861 (2012).

5 S. Ghosh, W. Bao, D. L. Nika, S. Subrina, E. P. Pokatilov, C. N. Lau, and A. A. Balandin, Nature Materials 9, 555 (2010).

D. L. Nika, S. Ghosh, E. P. Pokatilov, and A. A. Balandin, Applied Physics Letters 94, 203103 (2009).

K. H. Michel and B. Verberck, Physical Review B 78, 085424 (2008). J. Hu, S. Schiffli, A. Vallabhaneni, X. Ruan, and Y. P. Chen, Applied Physics Letters 97, 133107 (2010).

G. Fugallo, A. Cepellotti, L. Paulatto, M. Lazzeri, N. Marzari, and F. Mauri, Nano Letters 14, 6109 (2014).

D. L. Nika, E. P. Pokatilov, A. S. Askerov, and A. A. Balandin, Physical Review B 79, 155413 (2009).

E. Pop, V. Varshney, and A. K. Roy, MRS Bulletin 37, 1273 (2012).

A. A. Balandin, Nature Materials 10, 569 (2011).

I. Pletikosić, M. Kralj, P. Pervan, R. Brako, J. Coraux, A. T. N’Diaye, C. Busse, and T. Michely, Physical Review Letters 102, 056808 (2009).

D. C. Elias, R. V. Gorbachev, A. S. Mayorov, S. V. Morozov, A. A. Zhukov, P. Blake, L. A. Ponomarenko, I. V. Grigorieva, K. S. Novoselov, F. Guinea, and A. K. Geim, Nature Physics 7, 701 (2011). J.-K. Yu, S. Mitrovic, D. Tham, J. Varghese, and J. R. Heath, Nature Nanotechnology 5, 718 (2010).

16 K. Takeda and K. Shiraishi, Physical Review B 50, 14916 (1994). E. Durgun, S. Tongay, and S. Ciraci, Physical Review B 72, 075420 (2005).

S. Cahangirov, M. Topsakal, E. Aktürk, H. Şahin, and S. Ciraci, Physical Review Letters 102, 236804 (2009).

P. Vogt, P. De Padova, C. Quaresima, J. Avila, E. Frantzeskakis, M. C. Asensio, A. Resta, B. Ealet, and G. Le Lay, Physical Review Letters 108, 155501 (2012). B. Lalmi, H. Oughaddou, H. Enriquez, A. Kara, S. Vizzini, B. Ealet, and B. Aufray, Applied Physics Letters 97, 223109 (2010).

A. Kara, H. Enriquez, A. P. Seitsonen, L. C. Lew Yan Voon, S. Vizzini, B. Aufray, and H. Oughaddou, Surface Science Reports 67, 1 (2012). B. Feng, Z. Ding, S. Meng, Y. Yao, X. He, P. Cheng, L. Chen, and K. Wu, Nano Letters 12, 3507 (2012).

P. De Padova, O. Kubo, B. Olivieri, C. Quaresima, T. Nakayama, M. Aono, and G. Le Lay, Nano Letters 12, 5500 (2012).

W.-F. Tsai, C.-Y. Huang, T.-R. Chang, H. Lin, H.-T. Jeng, and A. Bansil, Nature Communications 4, 1500 (2013). 
N. D. Drummond, V. Zólyomi, and V. I. Fal'ko, Physical Review B 85, 075423 (2012).

Z. Ni, Q. Liu, K. Tang, J. Zheng, J. Zhou, R. Qin, Z. Gao, D. Yu, and J. Lu, Nano Letters 12, 113 (2012).

M. Hu, X. Zhang, and D. Poulikakos, Physical Review B 87, 195417 (2013).

Q.-X. Pei, Y.-W. Zhang, Z.-D. Sha, and V. B. Shenoy, Journal of Applied Physics 114, 033526 (2013).

Y. Du, J. Zhuang, H. Liu, X. Xu, S. Eilers, K. Wu, P. Cheng, J. Zhao, X. Pi, K.

W. See, G. Peleckis, X. Wang, and S. X. Dou, ACS Nano 8, 10019 (2014).

S. Z. Butler, S. M. Hollen, L. Cao, Y. Cui, J. A. Gupta, H. R. Gutiérrez, T. F.

Heinz, S. S. Hong, J. Huang, A. F. Ismach, E. Johnston-Halperin, M. Kuno, V. V. Plashnitsa, R. D. Robinson, R. S. Ruoff, S. Salahuddin, J. Shan, L. Shi, M. G. Spencer, M. Terrones, W. Windl, and J. E. Goldberger, ACS Nano 7, 2898 (2013).

X. Gu and R. Yang, Journal of Applied Physics 117, 025102 (2015).

X. Zhang, H. Xie, M. Hu, H. Bao, S. Yue, G. Qin, and G. Su, Physical Review B 89, 054310 (2014).

S. Chen, Q. Wu, C. Mishra, J. Kang, H. Zhang, K. Cho, W. Cai, A. A. Balandin, and R. S. Ruoff, Nature Materials 11, 203 (2012).

S. Broderick, U. Ray, S. Srinivasan, K. Rajan, and G. Balasubramanian, Applied Physics Letters 104, 243110 (2014).

F. Hao, D. Fang, and Z. Xu, Applied Physics Letters 99, 041901 (2011).

F. Leroy, J. Schulte, G. Balasubramanian, and M. C. Böhm, The Journal of

Chemical Physics 140, 144704 (2014).

U. Ray and G. Balasubramanian, Chemical Physics Letters 599, 154 (2014).

G. Audi, O. Bersillon, J. Blachot, and A. H. Wapstra, Nuclear Physics A 729, 3 (2003).

J. Tersoff, Physical Review B 39, 5566 (1989).

L. Lindsay and D. A. Broido, Physical Review B 81, 205441 (2010).

S. Plimpton, Journal of Computational Physics 117, 1 (1995).

W. Humphrey, Dalke, A. and Schulten, K., Journal of Molecular Graphics 14,, 33 (1996).

F. Müller-Plathe, The Journal of Chemical Physics 106, 6082 (1997).

A. Cao, Journal of Applied Physics 111, 083528 (2012).

G. Balasubramanian, I. K. Puri, M. C. Bohm, and F. Leroy, Nanoscale 3, 3714 (2011).

H. Zhang, G. Lee, and K. Cho, Physical Review B 84, 115460 (2011).

L. Lindsay, D. A. Broido, and N. Mingo, Physical Review B 82, 115427 (2010).

L. Lindsay, D. A. Broido, and N. Mingo, Physical Review B 83, 235428 (2011).

H. Xie, T. Ouyang, É. Germaneau, G. Qin, M. Hu, and H. Bao, Physical Review B 93, 075404 (2016).

Z. Guo, D. Zhang, and X.-G. Gong, Applied Physics Letters 95, 163103 (2009). 


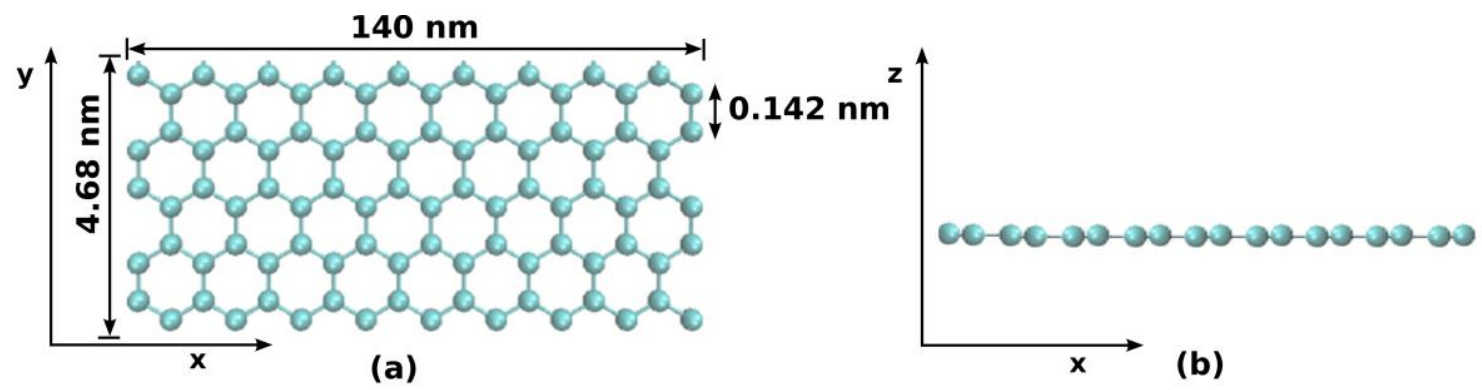

$134.7 \mathrm{~nm}$

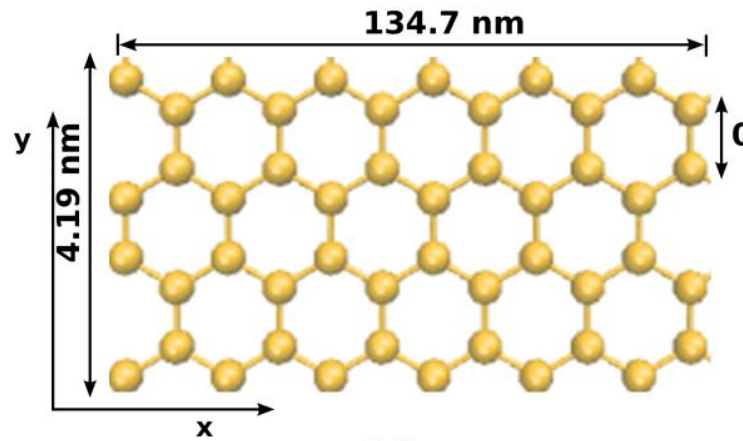

(c)

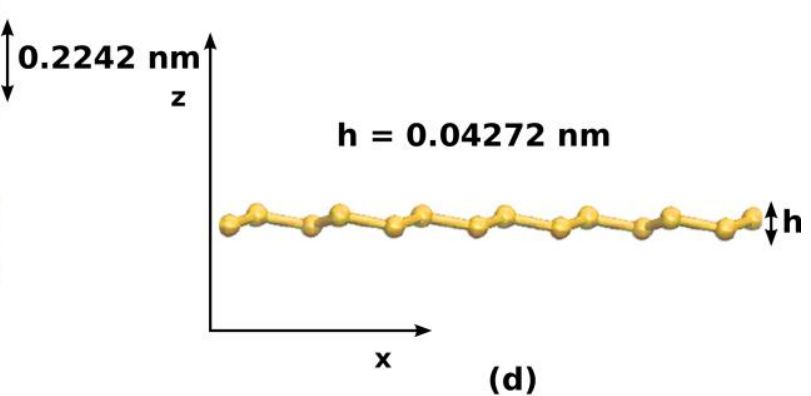

(d)

Figure 1. The equilibrated structure of graphene of length $L=140.24 \mathrm{~nm}$ and width $=4.32$ $\mathrm{nm}$ along the $x-y$ and $x-z$ planes is shown in (a) and (b) respectively. C-C bond length in graphene is $0.142 \mathrm{~nm}$. The view of the nanomaterial along the $x-z$ plane shows that graphene has no buckling in the structure and has an exact planar conformation. The equilibrated structure of silicene of length $L=134.70 \mathrm{~nm}$ and width $=4.19 \mathrm{~nm}$ along the $x$ $y$ and $x-z$ planes is shown in (c) and (d) respectively. Si-Si bond length in silicene is $0.2242 \mathrm{~nm}$. The view of the nanomaterial along the $x-z$ plane shows that silicene has regular undulations and is not exactly planar. The effective buckling height is computed to be $0.04272 \mathrm{~nm}$. 

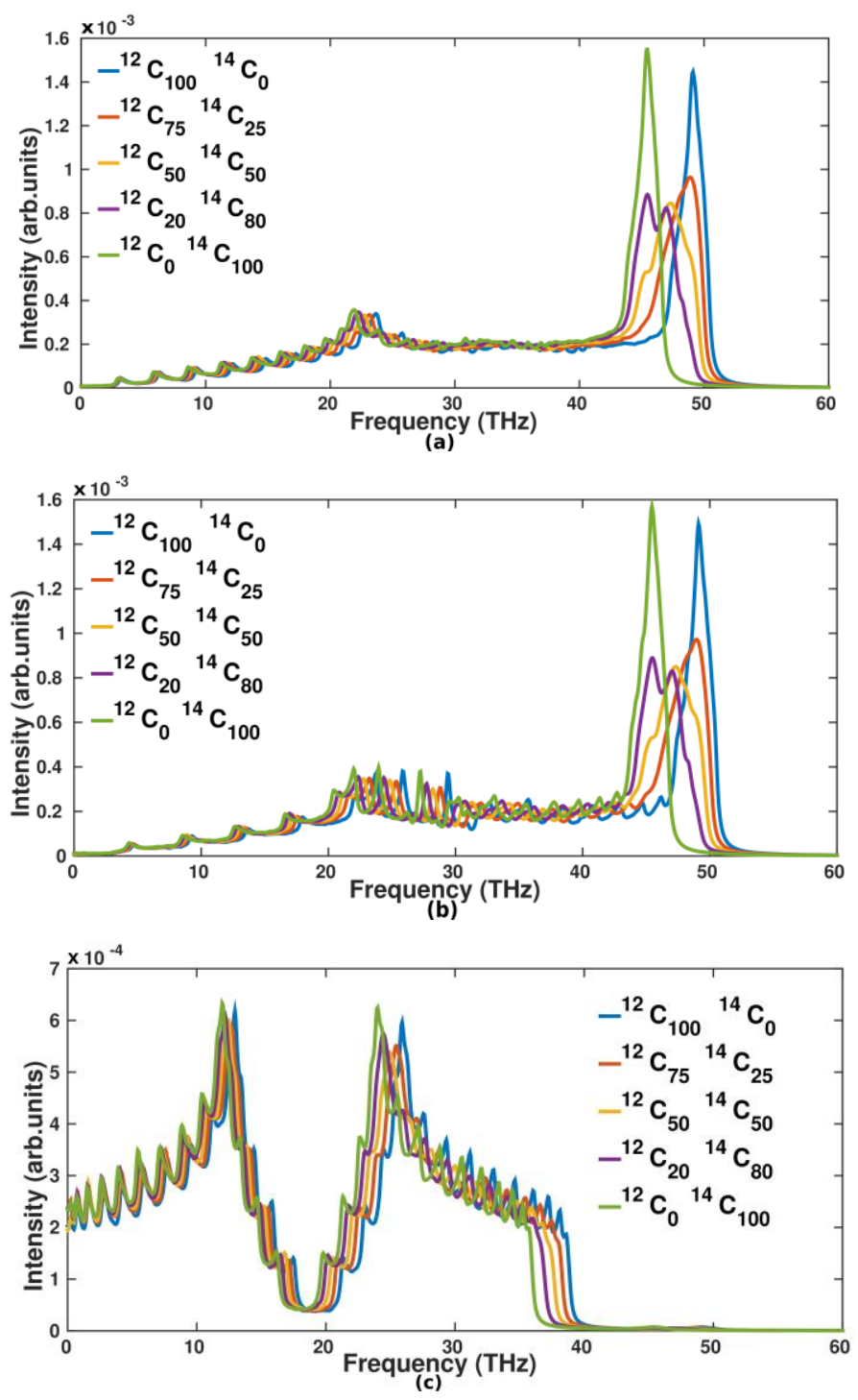

Figure 2. The phonon DOS for $0 \%$ (blue), 25\% (red), 50\% (yellow), 80\% (violet) and 100\% (green) isotope substituted graphene are presented: (a) in-plane longitudinal modes along the direction ( $x$-wise) of heat transfer, (b) in-plane transverse modes ( $y$-wise) and (c) out-of-plane flexural modes perpendicular ( $z$-wise) to the direction of heat transfer. The DOS intensity represented along the vertical axes is in arbitrary units. The legends represent each graphene structure as ${ }^{12} \mathrm{C}_{(100-100 \phi)}{ }^{14} \mathrm{C}_{100 \phi}$, where $\phi$ is the fraction of ${ }^{14} \mathrm{C}$ isotope. The phonon frequencies shift to lower values (redshift) for both the in-plane and out-of-plane vibrational modes. The low frequency modes that are observed along the $z-$ direction are delocalized. The intensity of the peaks decreases as mass disorder increases. 


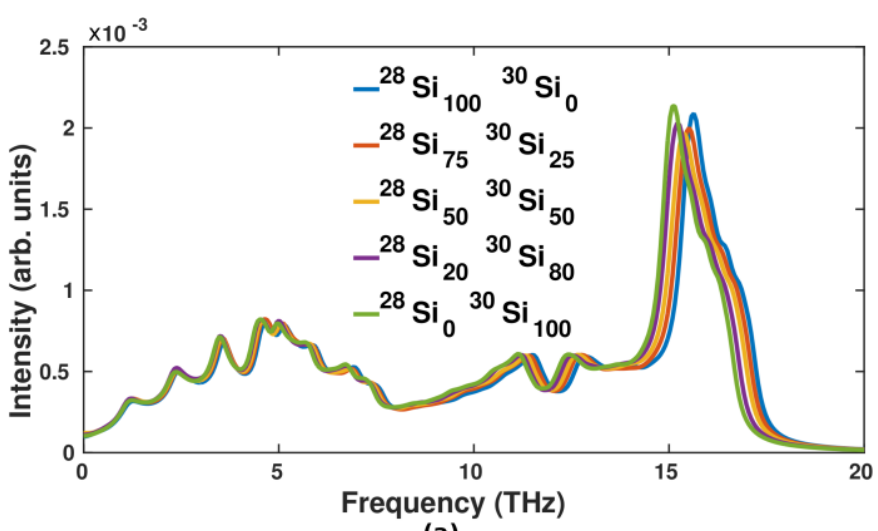

(a)
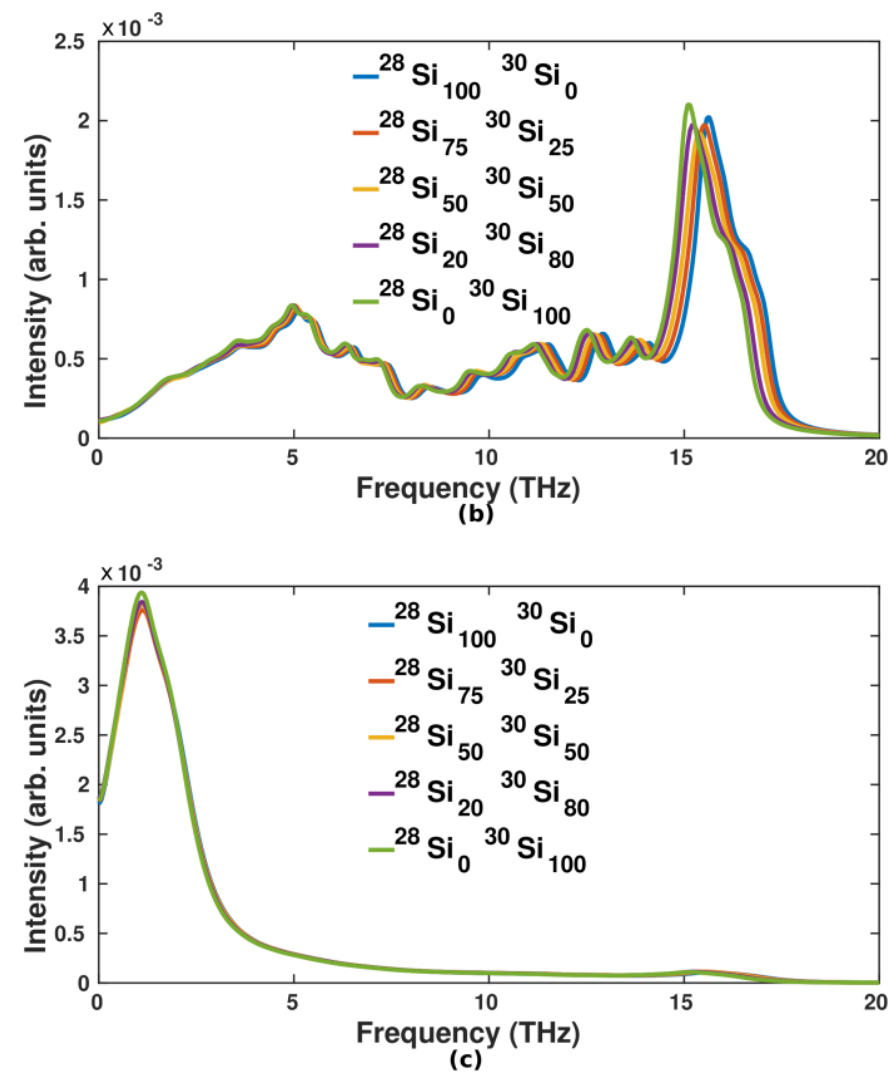

Figure 3. The phonon DOS for $0 \%$ (blue), $25 \%$ (red), $50 \%$ (yellow), $80 \%$ (violet) and $100 \%$ (green) isotope substituted silicene are presented: (a) in-plane longitudinal modes along the direction ( $x$-wise) of heat transfer, (b) in-plane transverse modes ( $y$-wise) and (c) out-of-plane modes perpendicular (z-wise) to the direction of heat transfer. The DOS intensity represented along the vertical axes is in arbitrary units. The legends represent each silicene structure as ${ }^{28} \mathrm{Si}_{(100-100 \phi)}{ }^{30} \mathrm{Si}_{100 \phi}$, where $\phi$ is the fraction of ${ }^{30} \mathrm{Si}$ isotope. The 
DOS spectra shifts to lower frequency values with the addition of isotopes and the intensity of the peaks decrease with increase in mass disorder, although these changes are less pronounced compared to that in graphene. Along $z$-direction, the phonon spectra show only a single significant peak around $1.2 \mathrm{THz}$. 


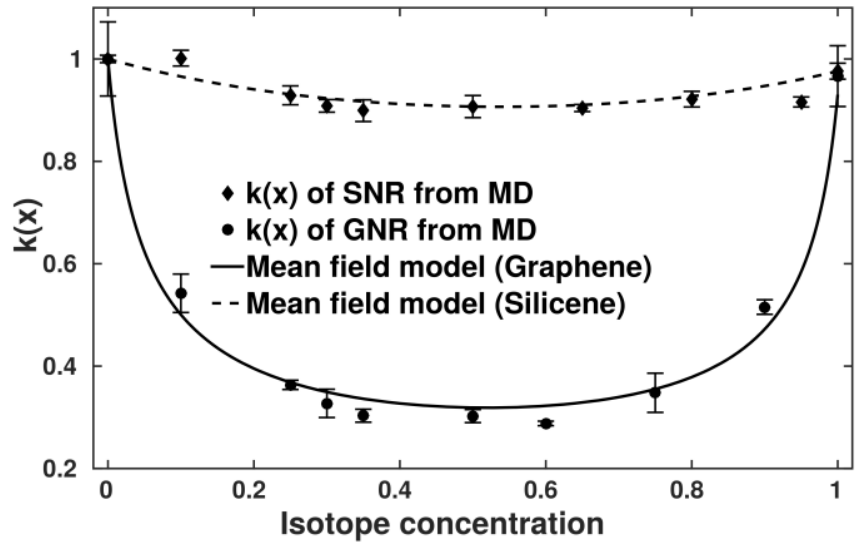

Figure 4. The variation of the thermal conductivity reduction $k(\phi)$ of mass disordered silicene and graphene is presented as the function of increasing isotope fractions $(\phi)$ from $0.0-1.0$ for nanomaterials simulated at $300 \mathrm{~K}$. The error bars represent the standard errors as derived from the computations. Thermal conductivity calculations from the MD simulations show reasonable agreement with the predictions of mean-field model given by equation (1) with appropriate correction for lattice anharmonicity, shown here as the dashed curve for silicene and the continuous curve for graphene. The value of the parameters, $\varepsilon$ and $M_{\alpha} / M_{\beta}$, are 0.0297 and 1.1655 for graphene and 0.7601 and 1.1297 for silicene. The model reproduces the sharp drop in $k$ for smaller isotope fractions $(<0.3)$ as well as the maximum reduction in $k$ at $50 \%$ isotope substitution in both the nanomaterials. 


\section{Graphical Abstract}

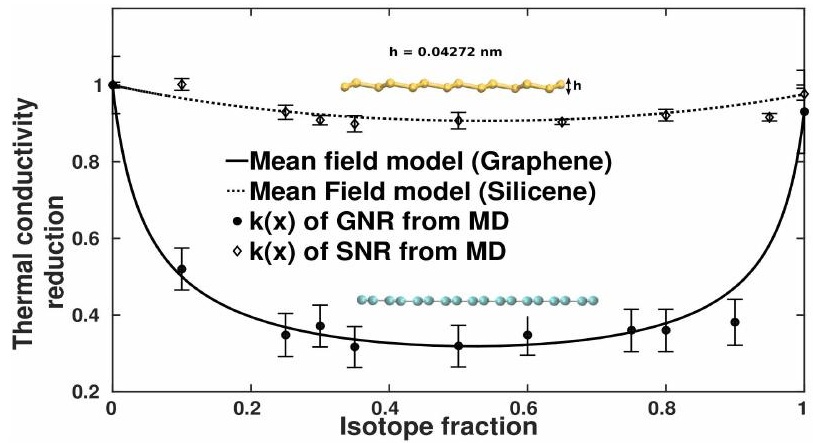

